Research Paper

\title{
miR-423 rs6505162 C>A polymorphism contributes to decreased Wilms tumor risk
}

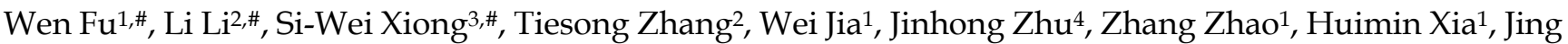 \\ $\mathrm{He}^{1, \otimes}$, Guo-Chang Liu ${ }^{1, \otimes}$ \\ 1. Department of Pediatric Surgery, Guangzhou Institute of Pediatrics, Guangzhou Women and Children's Medical Center, Guangzhou Medical University, \\ Guangzhou 510623, Guangdong, China \\ 2. Kunming Key Laboratory of Children Infection and Immunity, Yunnan Institute of Pediatrics, Kunming Children's Hospital, Kunming 650228, Yunnan, \\ China \\ 3. Department of Urology, Guangzhou First People's Hospital, the Second Affiliated Hospital of South China University of Technology, Guangzhou 510180, \\ Guangdong, China \\ 4. Department of Clinical Laboratory, Molecular Epidemiology Laboratory, Harbin Medical University Cancer Hospital, Harbin 150040, Heilongjiang, China \\ \#The first three authors contributed equally to this work.
}

$\triangle$ Corresponding authors: Guo-Chang Liu, Department of Pediatric Urology, Guangzhou Women and Children's Medical Center, Guangzhou Medical University, Guangzhou 510623, Guangdong, China, Email: starbless2003@126.com; or Jing He, Department of Pediatric Surgery, Guangzhou Institute of Pediatrics,Guangzhou Women and Children's Medical Center, Guangzhou Medical University, 9 Jinsui Road, Guangzhou 510623, Guangdong, China, Email: hejing198374@gmail.com or hejing@gwcmc.org.

(c) Ivyspring International Publisher. This is an open access article distributed under the terms of the Creative Commons Attribution (CC BY-NC) license (https://creativecommons.org/licenses/by-nc/4.0/). See http://ivyspring.com/terms for full terms and conditions.

Received: 2018.01.13; Accepted: 2018.04.14; Published: 2018.06.14

\begin{abstract}
Wilms tumor (WT) is the most prevalent urologic malignancy in childhood. Nonetheless, the genetic factors underlying WT remain largely unknown. The miR-423 rs6505162 C>A polymorphism is associated with the susceptibility to numerous cancers; however, no investigations have been conducted on its association with WT. To evaluate the correlation between the miR-423 rs6505162 C>A polymorphism and WT risk in Chinese children, we genotyped this polymorphism using the Taqman method in 145 cases and 531 cancer-free controls. Odds ratios (ORs) and $95 \%$ confidence intervals (Cls) were calculated to estimate the strength of the association. The results showed that the rs6505162 CA genotype was associated with decreased susceptibility to WT (CA versus CC: adjusted OR $=0.65,95 \%$ $\mathrm{Cl}=0.42-0.99, P=0.047)$. In the stratified analysis, we found that CA/AA genotypes conferred a significantly decreased overall risk of WT in children younger than 18 months (adjusted $O R=0.30,95 \%$ $\mathrm{Cl}=0.14-0.63, P=0.002$ ) and those with clinical stage $\mathrm{I}+\mathrm{II} W \mathrm{WT}$ (adjusted $\mathrm{OR}=0.42,95 \% \mathrm{Cl}=0.20-0.85$, $P=0.017$ ) when compared with $C C$ genotype. In summary, the miR-423 rs6505162 C>A polymorphism may negatively modify WT susceptibility in Chinese children. Our findings should be validated in larger studies involving other ethnicities.
\end{abstract}

Key words: Wilms tumor; miR-423; polymorphism; genetic susceptibility

\section{Introduction}

Wilms tumor (WT), also called nephroblastoma, morphologically resembles embryonic kidney cells. WT is a pediatric cancer that develops in young children, generally those younger than 5 years old [1]. It accounts for $8 \%$ of childhood malignancies and affects $1 / 10,000$ children. The tumor is considered a prototype of tumors resulting from abnormal differentiation and development of tissues [2]. Over the past decades, the treatment outcome has greatly improved, and the overall survival rate has reached
$90 \%$; however, a considerable portion of the suffers, including those with unfavourable histologic features, bilateral tumors and recurrent cases have survival rates less than $70 \%$. These higher risk groups compose $25 \%$ of WT patients $[3,4]$. Furthermore, treatment of WT comes at a cost, with up to $25 \%$ of the survivors reporting severe chronic health problems 25 years after diagnosis $[3,5,6]$. There remain intense clinical demands to find more effective therapies for high-risk subtypes and to minimize side effects later in life [7]. 
It has been demonstrated that mutations of the Wnt/ $\beta$-catenin pathway-related Wilms tumor gene 1 , $\beta$-catenin, and WTX account for approximately $1 / 3$ of WT cases. Extreme efforts are being made to find the genetic factors involved in the other $2 / 3$ of WT cases [2]. Some reports have noted that microRNA (miRNA) may play a key role in WT development, and $15 \%$ of WT cases habor mutations in the miRNA-processing genes $[2,4]$.

The abnormal expression of miR-423 (both mature forms of miR-423, which are called miR-423-3p and miR-423-5p) has been discovered in multiple cancers. The rs6505162 C>A polymorphism (nucleotide alteration from $\mathrm{C}$ to $\mathrm{A}$ ), mapping to 17q11.2, is located in pre-miR-423 [8-11]. An increasing number of studies have been conducted to estimate the association between the miR-423 rs6505162 C>A polymorphism and the risk of developing different cancers [12-19]. It has been found that the A allele of rs6505162 increases the risk of breast cancer [14, 15], but it decreases the risk of lung cancer and bladder cancer $[17,18]$. In a recent meta-analysis that included 17 eligible case-control studies, a significant association between miR-423 rs6505162 C>A and cancer susceptibility was observed in recessive models. Stratified analysis also revealed that those with the A allele had a significantly decreased risk of lung cancer [20].

Nucleotide polymorphism is highly likely to play different roles in different types of cancer depending on the inherent cancer-specific heterogeneity [21, 22]. Although it has been illustrated that rs6505162 is relevant to human cancers, the association between the miR-423 rs6505162 C>A polymorphism and WT susceptibility has not been demonstrated. Because of its implication in cancer, we hypothesized that this polymorphism might be able to modify WT susceptibility. Therefore, we performed a hospital-based case-control study with the purpose of determining whether this polymorphism is associated with WT risk in Chinese children.

\section{Materials and methods}

\section{Study subjects}

A total of 145 children who were diagnosed with histopathologically confirmed WT, according to the NWTS- 5 criteria, were included in this study. The diagnosis of this disease was based on both imaging and pathological examination. Tumor tissues were obtained from all cases by surgery or needle biopsy for pathological examination. All Chinese Han patients were recruited from the Guangzhou Women and Children's Medical Center, mainly between March 2001 and June 2016 [23-27]. In addition, 531 unrelated, age-, gender-, race-matched cancer-free volunteers recruited from the same medical centre were also enrolled [28-30]. Each individual donated 2 $\mathrm{ml}$ of blood for genomic DNA extraction. The response rate was nearly $90 \%$ and $95 \%$ for WT cases and cancer-free controls respectively. The investigation was conducted with the approval of the Institutional Review Board of the Guangzhou Women and Children's Medical Center. All the participants had written informed consent provided by their guardians.

\section{DNA extraction and genotyping}

We isolated total genomic DNA from peripheral blood leukocytes using the TIANamp Blood DNA Kit (TianGen Biotech Co., Ltd., Beijing, China) [28]. The miR-423 rs6505162 C>A polymorphism was genotyped using the TaqMan real-time PCR methodas described previously [31-33]. Genotyping was performed blind to the status of the case or control. Moreover, approximately $10 \%$ of samples were randomly selected to perform repeated assays, and the reproducibility was $100 \%$.

\section{Statistical analysis}

The frequency distribution of the polymorphism and the demographic variables between WT cases and controls were compared using the chi-squared test. Hardy-Weinberg equilibrium (HWE) in control subjects was tested by a goodness-of-fit chi-squared test. The association of the miR-423 rs6505162 C>A polymorphism with WT risk was assessed by calculating the odds ratios (ORs) and 95\% confidence intervals (CIs), using unconditional multivariate logistic regression analyses. Stratified analyses were performed for subgroups by age, gender and clinical stages. All statistical tests were two-sided. $P<0.05$ was considered as statistically significant. All statistical analyses were performed using SAS software (Version 9.4; SAS Institute, Cary, NC, USA).

\section{Results}

\section{Demographic characteristics}

As shown in Supplemental Table 1, no striking differences in age or gender between the two groups $[25,26]$. There were $4(2.76 \%), 49(33.79 \%), 50$ $(34.48 \%)$, and $33(22.76 \%)$ individuals with clinical stage I, II, III, and IV Wilms tumor, respectively, according to the NWTS-5 criteria [34].

\section{Association between miR-423 rs6505162 C>A polymorphism and WT susceptibility}

The genotype distributions of the miR-423 rs6505162 C $>$ A polymorphism in WT cases and cancer-free controls are shown in Table 1. A total of 145 cases and 530 controls were successfully 
genotyped. Genotype analysis for the miR-423 rs6505162 C>A polymorphism suggested that there was no significant deviation from HWE in the control group $(P=0.576)$. We observed that the rs6505162 CA genotype had decreased risk of WT at an adjusted OR of $0.65(95 \% \mathrm{CI}=0.42-0.99, P=0.047)$ when compared to the CC genotype. No significant associations were observed for other models (AA vs. CC: adjusted $\mathrm{OR}=0.91,95 \% \mathrm{CI}=0.33-2.50 ; \mathrm{AA} / \mathrm{CA}$ vs. $\mathrm{CC}$ : adjusted $\mathrm{OR}=0.67,95 \% \mathrm{CI}=0.45-1.01 ; \mathrm{AA}$ vs. $\mathrm{CC} / \mathrm{CA}$ : adjusted $\mathrm{OR}=1.02,95 \% \mathrm{CI}=0.37-2.81$; and A vs. $\mathrm{C}$ : adjusted $\mathrm{OR}=0.75,95 \% \mathrm{CI}=0.52-1.06$ ).

\section{Stratified analysis}

We further observed the association between the miR-423 rs6505162 C>A polymorphism with WT risk in the stratified analysis by age, gender, and clinical stages (Table 2). We found that carriers of CA/AA genotypes had significantly decreased overall risk of WT in children younger than 18 months (adjusted $\mathrm{OR}=0.30,95 \% \mathrm{CI}=0.14-0.63, P=0.002)$ and clinical stage
I+II WT (adjusted OR=0.42, 95\% CI $=0.20-0.85$, $P=0.017)$ compared with CC genotype carriers. We also found a borderline association between a decreased WT risk and the CA/AAgenotypes in females (adjusted OR $=0.57,95 \%$ CI $=0.31-1.07$, $P=0.081$ ).

When false-positive report probability analysis was performed, the noteworthy findings disappeared at the prior probability level of 0.1 and FPRP threshold of 0.2 (Table 3 ).

\section{Discussion}

In this hospital-based case-control study, we explored the correlation between the miR-423 rs6505162 C>A polymorphism and WT susceptibility and subsequently analysed the effects of combinations of this polymorphism and clinical features. It has been identified that the miR-423 rs6505162 C>A polymorphism is associated with WT risk in a Chinese population.

Table 1. Genotype distributions of miR-423 rs6505162 C>A polymorphism and Wilms tumor susceptibility.

\begin{tabular}{|c|c|c|c|c|c|c|c|}
\hline Genotype & Cases $(\mathrm{N}=145)$ & Controls $(\mathrm{N}=530)$ & $P$ a & Crude OR (95\% CI) & $P$ & Adjusted OR $(95 \% \mathrm{CI}){ }^{\mathrm{b}}$ & $P \mathrm{~b}$ \\
\hline \multicolumn{8}{|c|}{ rs6505162 (HWE=0.576) } \\
\hline $\mathrm{CC}$ & $106(73.10)$ & $342(64.53)$ & & 1.00 & & 1.00 & \\
\hline CA & $34(23.45)$ & $170(32.08)$ & & $0.65(0.42-0.99)$ & 0.045 & $0.65(0.42-0.99)$ & 0.047 \\
\hline AA & $5(3.45)$ & $18(3.40)$ & & $0.90(0.33-2.47)$ & 0.832 & $0.91(0.33-2.50)$ & 0.847 \\
\hline Additive & & & 0.131 & $0.74(0.52-1.06)$ & 0.099 & $0.74(0.52-1.06)$ & 0.104 \\
\hline Dominant & $39(26.90)$ & $188(35.47)$ & 0.053 & $0.67(0.45-1.01)$ & 0.054 & $0.67(0.45-1.01)$ & 0.057 \\
\hline Recessive & $140(96.55)$ & $512(96.60)$ & 0.976 & $1.02(0.37-2.79)$ & 0.976 & $1.02(0.37-2.81)$ & 0.963 \\
\hline $\mathrm{C}$ & $246(84.83)$ & $854(80.57)$ & & 1.00 & & 1.00 & \\
\hline A & $44(15.17)$ & 206 (19.43) & 0.098 & $0.74(0.52-1.06)$ & 0.099 & $0.75(0.52-1.06)$ & 0.104 \\
\hline
\end{tabular}

Table 2. Stratification analysis for the association between miR-423 rs6505162 C>A polymorphism and Wilms tumor risk.

\begin{tabular}{|c|c|c|c|c|c|c|}
\hline Variables & $\mathrm{CC}$ & $\mathrm{CA} / \mathrm{AA}$ & Crude OR & $P$ & Adjusted OR a & $P$ a \\
\hline & \multicolumn{2}{|c|}{ (Cases/Controls) } & $(95 \% \mathrm{CI})$ & & $(95 \% \mathrm{CI})$ & \\
\hline \multicolumn{7}{|c|}{ Age, month } \\
\hline$\leq 18$ & $57 / 152$ & $9 / 81$ & $0.30(0.14-0.63)$ & 0.002 & $0.30(0.14-0.63)$ & 0.002 \\
\hline$>18$ & $49 / 190$ & $30 / 107$ & $1.09(0.65-1.82)$ & 0.749 & $1.09(0.65-1.81)$ & 0.754 \\
\hline \multicolumn{7}{|l|}{ Gender } \\
\hline Females & $48 / 147$ & $16 / 86$ & $0.57(0.31-1.07)$ & 0.078 & $0.57(0.31-1.07)$ & 0.081 \\
\hline Males & $58 / 195$ & $23 / 102$ & $0.76(0.44-1.30)$ & 0.314 & $0.76(0.44-1.30)$ & 0.316 \\
\hline \multicolumn{7}{|c|}{ Clinical stage } \\
\hline $\mathrm{I}+\mathrm{II}$ & $43 / 342$ & $10 / 188$ & $0.42(0.21-0.86)$ & 0.018 & $0.42(0.20-0.85)$ & 0.017 \\
\hline $\mathrm{III}+\mathrm{IV}$ & $56 / 342$ & $27 / 188$ & $0.88(0.54-1.44)$ & 0.602 & $0.88(0.54-1.44)$ & 0.609 \\
\hline
\end{tabular}

OR, odds ratio; $\mathrm{CI}$, confidence interval.

a Adjusted for age and gender.

Table 3. False-positive report probability analysis for the significant findings between miR-423 rs6505162 C>A polymorphism and Wilms tumor risk.

\begin{tabular}{|c|c|c|c|c|c|c|c|c|}
\hline \multirow[t]{2}{*}{ Genotype } & \multirow[t]{2}{*}{ Crude OR (95\% CI) } & \multirow[t]{2}{*}{$P$ a } & \multirow[t]{2}{*}{ Statistical power ${ }^{b}$} & \multicolumn{5}{|c|}{ Prior probability } \\
\hline & & & & 0.25 & 0.1 & 0.01 & 0.001 & 0.0001 \\
\hline CA vs. CC & $0.65(0.42-0.99)$ & 0.045 & 0.442 & 0.234 & 0.478 & 0.910 & 0.990 & 0.999 \\
\hline \multicolumn{9}{|c|}{$\mathrm{CA} / \mathrm{AA}$ vs. CC } \\
\hline$\leq 18$ & $0.30(0.14-0.63)$ & 0.002 & 0.024 & 0.157 & 0.358 & 0.860 & 0.984 & 0.998 \\
\hline $\mathrm{I}+\mathrm{II}$ & $0.42(0.21-0.86)$ & 0.018 & 0.116 & 0.315 & 0.580 & 0.938 & 0.994 & 0.999 \\
\hline
\end{tabular}


MiRNAs are a category of single-stranded, non-coding, endogenous RNAs approximately 22 nucleotides in length. They guide RNA-induced silencing complexes to the miRNA recognition elements of the targeted protein-coding transcripts or other competitive endogenous RNAs, and thus play a role in post transcriptional control $[35,36]$. There are approximated to be at least 300 miRNAs (and there might be as many as 1000) in the human genome, which makes miRNAs one of the largest categories of gene regulator [37]. It has been reported that over $1 / 2$ of miRNAs are located in cancer-associated genomic regions or fragile sites and are involved in tumorigenesis as suppressors or oncogenes [38]. The aberrant expression of different miRNAs in cancer development has been observed [37]. It is reasonable to suppose that miRNA expression and maturation might be altered by single nucleotide polymorphisms in miRNAs. These polymorphisms could influence the effects of miRNAs on their target genes, potentially leading to aberrant metabolism and modified cancer susceptibility [38-40].

As reported before, rs6505162 is located in the first intron of the nuclear speckle splicing regulatory protein (NSRP1) gene [15], which produces two mature transcripts called miR-423-3p and miR-423-5p [41]. The abnormal expression of miR-423 has been discovered in multiple cancers, but the potential role of miR-423 in cancer is complicated and varies in different cancer types. Different expression patterns of miR-423 have been found; for example, miR-423 is under-expressed in oral cancer [42] but over-expressed in head and neck cancer [43]. MiR-423 acts as a tumor suppressor in some tumors [42], but as an oncogene in the other ones [44]. In addition, it has been reported that the rs6505162 C $>$ A polymorphism promotes mature miR-423 expression in cell lines from breast cancer and endometrial carcinoma [45, 46]. It is still ambiguous whether modulations of mature levels of miR-423 arerelated to the involvement of this polymorphism in many other cancers [38]. All these inconsistent results remind us of the significant heterogeneity among cancer types [38].

Considering the importance of the miR-423 rs6505162 C>A polymorphism in carcinogenesis, we assessed the association of the miR-423 rs6505162 C>A polymorphism with WT risk. To the best of our knowledge, this is the first study to verify the association between the miR-423 rs6505162 C>A polymorphism and pediatric tumor susceptibility. Also, there is no other study investigated the association between other polymorphisms within pre-miRNAs and WT risk. We found that the rs6505162 CA genotype was associated with a decreased susceptibility for WT. The rs6505162 polymorphism lies within an intron of the NSRP1gene, and it is predicted by SNPinfo (http://snpinfo.niehs.nih.gov/) software to be a transcription factor binding site. The $\mathrm{C}>\mathrm{A}$ alteration may cause alteration in binding ability and downstream gene expression, and sequentially modify WT susceptibility. In the subgroup analysis, the CA/AA genotype was more common among children younger than 18 months and with clinical stage I+II tumors, which may be ascribed to genetic susceptibility as well as limited sample size. In summary, these results indicated that the miR-423 rs6505162 C>A polymorphism may modify WT susceptibility in Southern Chinese children. Further functional studies are needed to investigate the specific mechanisms by which this polymorphism modifies cancer risk.

Although this is the first study to assess the correlation between the miR-423 rs6505162 C>A polymorphism and WT risk, several limitations should be addressed. First, it may have limited statistical power because of the relatively small sample size. Only 145 cases and 531 controls were included in this study due to the low occurrence rate of WT. Thus, statistic power of this study was compromised and the noteworthy findings might be chance observations (FPRP values larger than 0.2 at the prior probability level of 0.1 ). Second, selection and information bias might be unavoidable because of the nature of a retrospective study design, which limited our ability to correlate gene-environmental interactions with WT susceptibility. Finally, we conducted a case-control designed study to evaluate the association of the miR-423 rs6505162 C>A polymorphism with WT risk, and we have not explored the potential mechanisms of this polymorphism in cell lines, which should be studied in the future.

In conclusion, in this study, we found that the miR-423 rs6505162 C>A polymorphism may be associated with a decreased WT susceptibility in a Chinese population. However, well-designed prospective studies with larger sample sizes, different ethnicities, and more polymorphisms, as well as further functional studies are needed to confirm our findings.

\section{Acknowledgments}

This study was supported by grants from the Science and Technology Project of Guangzhou (No: 201804010037), the Pearl River S\&T Nova Program of Guangzhou (No: 201710010086) and the State Clinical Key Specialty Construction Project (Paediatric Surgery) 2013 (No: GJLCZD1301). 


\section{Abbreviations}

WT: Wilms tumor; miRNA: microRNA; HWE: Hardy-Weinberg equilibrium; OR: odds ratio; CI: confidence interval.

\section{Supplementary Material}

Supplementary table. http://www.jcancer.org/v09p2460s1.pdf

\section{Competing Interests}

The authors have declared that no competing interest exists.

\section{References}

1. Hohenstein $P$, Pritchard-Jones $K$, Charlton J. The yin and yang of kidney development and Wilms' tumors. Genes \& development. 2015; 29: 467-82.

2. Tian F, Yourek G, Shi X, Yang Y. The development of Wilms tumor: from WT1 and microRNA to animal models. Biochimica et biophysica acta. 2014; 1846: 180-7.

3. Dome JS, Fernandez CV, Mullen EA, Kalapurakal JA, Geller JI, Huff V, et al. Children's Oncology Group's 2013 blueprint for research: renal tumors. Pediatric blood \& cancer. 2013; 60: 994-1000.

4. Dome JS, Graf N, Geller JI, Fernandez CV, Mullen EA, Spreafico F, et al. Advances in Wilms Tumor Treatment and Biology: Progress Through International Collaboration. Journal of clinical oncology : official journal of the American Society of Clinical Oncology. 2015; 33: 2999-3007.

5. van Waas $M$, Neggers SJ, Raat $H$, van Rij CM, Pieters $R$, van den Heuvel-Eibrink MM. Abdominal radiotherapy: a major determinant of metabolic syndrome in nephroblastoma and neuroblastoma survivors. PloS one. 2012; 7: e52237.

6. Termuhlen AM, Tersak JM, Liu Q, Yasui Y, Stovall M, Weathers R, et al. Twenty-five year follow-up of childhood Wilms tumor: a report from the Childhood Cancer Survivor Study. Pediatric blood \& cancer. 2011; 57: 1210-6.

7. Perotti D, Hohenstein P, Bongarzone I, Maschietto M, Weeks M, Radice $\mathrm{P}$, et al. Is Wilms tumor a candidate neoplasia for treatment with WNT/beta-catenin pathway modulators?--A report from the renal tumors biology-driven drug development workshop. Molecular cancer therapeutics. 2013; 12: 2619-27.

8. Guled M, Lahti L, Lindholm PM, Salmenkivi K, Bagwan I, Nicholson AG, et al. CDKN2A, NF2, and JUN Are Dysregulated Among Other Genes by miRNAs in Malignant Mesothelioma-A miRNA Microarray Analysis. Gene Chromosome Canc. 2009; 48: 615-23.

9. Hui ABY, Lenarduzzi M, Krushel T, Waldron L, Pintilie M, Shi W, et al. Comprehensive MicroRNA Profiling for Head and Neck Squamous Cell Carcinomas. Clin Cancer Res. 2010; 16: 1129-39.

10. Farazi TA, Horlings HM, Ten Hoeve JJ, Mihailovic A, Halfwerk H, Morozov $\mathrm{P}$, et al. MicroRNA sequence and expression analysis in breast tumors by deep sequencing. Cancer research. 2011; 71: 4443-53.

11. Lerman G, Avivi C, Mardoukh C, Barzilai A, Tessone A, Gradus B, et al. MiRNA expression in psoriatic skin: reciprocal regulation of hsa-miR-99a and IGF-1R. PloS one. 2011; 6: e20916.

12. He $B$, Pan $Y, X u Y$, Deng $Q$, Sun $H$, Gao $T$, et al. Associations of polymorphisms in microRNAs with female breast cancer risk in Chinese population. Tumour biology : the journal of the International Society for Oncodevelopmental Biology and Medicine. 2015; 36: 4575-82.

13. Ma Y, Wang R, Zhang J, Li W, Gao C, Liu J, et al. Identification of miR-423 and miR-499 polymorphisms on affecting the risk of hepatocellular carcinoma in a large-scale population. Genetic testing and molecular biomarkers. 2014; 18: 516-24

14. Morales S, Gulppi F, Gonzalez-Hormazabal P, Fernandez-Ramires R, Bravo T, Reyes JM, et al. Association of single nucleotide polymorphisms in Pre-miR-27a, Pre-miR-196a2, Pre-miR-423, miR-608 and Pre-miR-618 with breast cancer susceptibility in a South American population. BMC genetics. 2016; 17: 109.

15. Smith RA, Jedlinski DJ, Gabrovska PN, Weinstein SR, Haupt L, Griffiths LR. A Genetic Variant Located in miR-423 is Associated with Reduced Breast Cancer Risk. Cancer Genom Proteom. 2012; 9: 115-8.

16. Umar M, Upadhyay R, Prakash G, Kumar S, Ghoshal UC, Mittal B. Evaluation of Common Genetic Variants in Pre-microRNA in
Susceptibility and Prognosis of Esophageal Cancer. Mol Carcinogen. 2013; 52: 10-8

17. Yin ZH, Cui ZG, Ren YW, Xia LZ, Wang QQ, Zhang Y, et al. Association between polymorphisms in pre-miRNA genes and risk of lung cancer in a Chinese non-smoking female population. Lung Cancer. 2016; 94: 15-21.

18. Yang H, Dinney CP, Ye Y, Zhu Y, Grossman HB, Wu X. Evaluation of genetic variants in microRNA-related genes and risk of bladder cancer. Cancer research. 2008; 68: 2530-7.

19. He J, Zou Y, Liu X, Zhu J, Zhang J, Zhang R, et al. Association of common genetic variants in pre-microRNAs and neuroblastoma susceptibility: a two-center study in Chinese children. Molecular therapy Nucleic acids. 2018. DOI: 10.1016/j.omtn.2018.01.003.

20. Chen R, Zheng Y, Zhuo L, Wang S. The association between miR-423 rs6505162 polymorphism and cancer susceptibility: a systematic review and meta-analysis. Oncotarget. 2017; 8: 40204-13.

21. Tan H, Bao J, Zhou X. Genome-wide mutational spectra analysis reveals significant cancer-specific heterogeneity. Scientific reports. 2015; 5: 12566.

22. Tan H. The association between gene SNPs and cancer predisposition: Correlation or causality? EBioMedicine. 2017; 16: 8-9.

23. Liang X, Lan C, Jiao G, Fu W, Long X, An Y, et al. Therapeutic inhibition of SGK1 suppresses colorectal cancer. Experimental \& molecular medicine. 2017; 49: e399.

24. Chen W, Shui F, Liu C, Zhou X, Li W, Zheng Z, et al. Corrigendum: Novel Peripherally Restricted Cannabinoid 1 Receptor Selective Antagonist TXX-522 with Prominent Weight-Loss Efficacy in Diet Induced Obese Mice. Frontiers in pharmacology. 2017; 8: 890.

25. Fu W, Zhuo ZJ, Jia W, Zhu JH, Zhu SB, Lin ZF, et al. Association between TP53 gene Arg72Pro polymorphism and Wilms' tumor risk in a Chinese population. Oncotargets Ther. 2017; 10: 1149-54.

26. Liu GC, Zhuo ZJ, Zhu SB, Zhu JH, Jia W, Zhao Z, et al. Associations between LMO1 gene polymorphisms and Wilms' tumor susceptibility. Oncotarget. 2017; 8: 50665-72.

27. Bassig BA, Dai $Y$, Vermeulen $R$, Ren $D, H u ~ W$, Duan $H$, et al. Occupational exposure to diesel engine exhaust and alterations in immune/inflammatory markers: a cross-sectional molecular epidemiology study in China. Carcinogenesis. 2017; 38: 1104-11.

28. He J, Zhang R, Zou Y, Zhu J, Yang T, Wang F, et al. Evaluation of GWAS-identified SNPs at $6 \mathrm{p} 22$ with neuroblastoma susceptibility in a Chinese population. Tumour biology : the journal of the International Society for Oncodevelopmental Biology and Medicine. 2016; 37: 1635-9.

29. He J, Wang F, Zhu J, Zhang Z, Zou Y, Zhang R, et al. The TP53 gene rs1042522 C>G polymorphism and neuroblastoma risk in Chinese children. Aging. 2017; 9: 852-9.

30. He J, Zou Y, Wang T, Zhang R, Yang T, Zhu J, et al. Genetic Variations of GWAS-Identified Genes and Neuroblastoma Susceptibility: a Replication Study in Southern Chinese Children. Translational oncology. 2017; 10: 936-41.

31. Su P, Fu W, Yao H, Liu L, Ding D, Feng F, et al. Enhanced photovoltaic properties of perovskite solar cells by $\mathrm{TiO} 2$ homogeneous hybrid structure. Royal Society open science. 2017; 4: 170942.

32. Lou J, Gong J, Ke J, Tian J, Zhang Y, Li J, et al. A functional polymorphism located at transcription factor binding sites, rs6695837 near LAMC1 gene, confers risk of colorectal cancer in Chinese populations. Carcinogenesis. 2017; 38: 177-83.

33. He J, Qiu LX, Wang MY, Hua RX, Zhang RX, Yu HP, et al. Polymorphisms in the XPG gene and risk of gastric cancer in Chinese populations. Human genetics. 2012; 131: 1235-44.

34. Beckwith JB. National Wilms Tumor Study: an update for pathologists. Pediatric and developmental pathology : the official journal of the Society for Pediatric Pathology and the Paediatric Pathology Society. 1998; 1: 79-84.

35. Bartel DP. MicroRNAs: target recognition and regulatory functions. Cell. 2009; 136: 215-33

36. Filipowicz W, Bhattacharyya SN, Sonenberg N. Mechanisms of post-transcriptional regulation by microRNAs: are the answers in sight? Nature reviews Genetics. 2008; 9: 102-14

37. Esquela-Kerscher A, Slack FJ. Oncomirs - microRNAs with a role in cancer. Nature reviews Cancer. 2006; 6: 259-69.

38. Ryan BM, Robles AI, Harris CC. Genetic variation in microRNA networks: the implications for cancer research. Nature reviews Cancer. 2010; 10: 389-402.

39. Kumar MS, Lu J, Mercer KL, Golub TR, Jacks T. Impaired microRNA processing enhances cellular transformation and tumorigenesis. Nature genetics. 2007; 39: 673-7.

40. Nicoloso MS, Sun H, Spizzo R, Kim H, Wickramasinghe P, Shimizu M, et al. Single-Nucleotide Polymorphisms Inside MicroRNA Target Sites Influence Tumor Susceptibility. Cancer research. 2010; 70: 2789-98. 
41. Afanasyeva EA, Hotz-Wagenblatt A, Glatting KH, Westermann F. New miRNAs cloned from neuroblastoma. BMC genomics. 2008; 9: 52.

42. Roy R, Singh R, Chattopadhyay E, Ray A, Sarkar N, Aich R, et al. MicroRNA and target gene expression based clustering of oral cancer, precancer and normal tissues. Gene. 2016; 593: 58-63.

43. Hui AB, Lenarduzzi M, Krushel T, Waldron L, Pintilie M, Shi W, et al. Comprehensive MicroRNA profiling for head and neck squamous cell carcinomas. Clin Cancer Res. 2010; 16: 1129-39.

44. Lin J, Huang S, Wu S, Ding J, Zhao Y, Liang L, et al. MicroRNA-423 promotes cell growth and regulates $G(1) / S$ transition by targeting p21Cip1/Waf1 in hepatocellular carcinoma. Carcinogenesis. 2011; 32: 1641-7.

45. Zhao H, Gao A, Zhang Z, Tian R, Luo A, Li M, et al. Genetic analysis and preliminary function study of miR-423 in breast cancer. Tumour biology : the journal of the International Society for Oncodevelopmental Biology and Medicine. 2015; 36: 4763-71.

46. Su X, Hu Y, Li Y, Cao JL, Wang XQ, Ma X, et al. The polymorphism of rs6505162 in the MIR423 coding region and recurrent pregnancy loss. Reproduction. 2015; 150: 65-76. 